Patricia Heller (Orcid: 0000-0002-5102-2440) ${ }^{1}$ Antuani Rafael Baptistella (Orcid: 0000-0003-1708-9921) ${ }^{1}$ Elcio Luiz Bonamigo (Orcid: 0000-0002-0226-7070)

\section{CONFLITOS MORAIS NA PRÁXIS DO ENSINO \\ DA FISIOTERAPIA: PERCEPÇÃO E ATITUDE DE PACIENTES}

\author{
MORAL CONFLICTS IN THE PHYSIOTHERAPY \\ PRAXIS TEACHING: PATIENTS' PERCEPTION AND \\ ATTITUDE
}

\begin{abstract}
RESUMO
Objetivo: Os pacientes contribuem para o aprendizado dos estudantes, porém, na tríplice relação entre pacientes, fisioterapeutas e estudantes no âmbito do ensino, podem surgir problemas éticos. O objetivo desta pesquisa foi analisar a percepção dos pacientes atendidos por estudantes do curso de graduação em fisioterapia de uma universidade acerca de problemas éticos que surgem na práxis da fisioterapia. Realizou-se pesquisa descritiva transversal, com abordagem quantitativa por meio de questionário estruturado aplicado aos pacientes atendidos nos estágios curriculares da instituição de ensino. Os pacientes relataram estar muito satisfeitos com o atendimento realizado com a participação de acadêmicos $(98,3 \%)$ porque precisam cuidar da saúde; e os alunos, aprender (51,3\%). Verificou-se que somente alguns acadêmicos não pedem consentimento aos pacientes para o atendimento $(6 \%)$ ou os tratam com desrespeito $(5,9 \%)$. Entretanto, alguns pacientes disseram deixar de comunicar algum problema de saúde por constrangimento pela presença de alunos (14,5\%) ou manifestaram temor pela possibilidade de quebra do sigilo profissional (6\%). Conclui-se que os pacientes estão satisfeitos tanto pelo atendimento recebido com a participação de acadêmicos como em contribuir para seu aprendizado, e que os problemas morais que integram essa prática fisioterapêutica no âmbito do ensino são raros. Infere-se que a abordagem desses temas durante o ensino da graduação e no teor do Código de Ética e Deontologia da Fisioterapia poderá contribuir para que a formação do fisioterapeuta seja mais ainda ética e humana, voltada ao cuidado e garantindo uma boa relação terapeuta/paciente.
\end{abstract}

Autor Correspondente

elcio.bonamigo@unoesc.edu.br

${ }^{1}$ Programa de Mestrado em Biociências e Saúde. Universidade do Oeste de Santa Catarina
Palavras-chave: Satisfação do paciente. Educação. Fisioterapia. Bioética. Ética profissional.

\begin{abstract}
Objective: Patients contribute to students' learning, but in this triple relationship between physiotherapists, patients and students in the field of education, ethical problems can arise. The objective of this research was to analyze the perception of the patients attended by students of the undergraduate course in physiotherapy of a University about ethical problems that arise in the praxis of physiotherapy. Descriptive cross-sectional research, with a quantitative approach through a structured questionnaire applied to the patients attending the curricular stages of the educational institution. Patients reported being very satisfied with the attendance performed with the participation of academics (98.3\%) because they need to take care of their health and the students need to learn (51.3\%). It was verified that only a few academics do not ask patients to consent for their care (6\%) or treat them with disrespect (5.9\%). However, some patients said they did not report any health problems due to the presence of students (14.5\%) or expressed fear of a possible breach of professional secrecy (6\%). It is concluded that patients are satisfied both with the care received and in contributing to the teaching of academics and the ethical problems that integrate the physiotherapeutic practice in teaching are rare. It is inferred that the approach of these subjects during undergraduate education and in the content of the Code of Ethics and Deontology of Physiotherapy may contribute to the formation of the physiotherapist that is more ethical and human, focused on care and ensuring a good therapist / patient relationship.
\end{abstract}

Keywords: Patient satisfaction. Education. Physical Therapy Specialty. Bioethics. Ethics, professional. 


\section{INTRODUÇÃO}

O surgimento de novos cursos de fisioterapia no Brasil e no mundo suscitou a necessidade de inclusão de maior número de pacientes no processo ensino-aprendizagem. $\mathrm{O}$ contato direto com pacientes durante a graduação, sob o acompanhamento de professores, oportuniza aos alunos a vivência de situações práticas imprescindíveis para o aprimoramento de sua formação científica e humanística ${ }^{1}$.

A presença de estudantes durante a assistência fisioterápica poderia ser interpretada como uma interferência na privacidade dos pacientes, acarretando dúvidas sobre sua aceitação. Contudo, pesquisa realizada com pacientes atendidos em outra profissão da saúde mostrou o contrário, já que $98 \%$ dos participantes concordavam com a presença de acadêmicos e tinham satisfação em contribuir para sua formação ${ }^{2}$.

A inclusão de estudantes durante $\mathrm{o}$ atendimento fisioterapêutico pode concorrer para o melhor acolhimento dos pacientes mesmo que, em algumas situações, a exposição de parte(s) do seu corpo a um maior número de pessoas possa gerar algum constrangimento. Entretanto, a gratuidade nos atendimentos e a oportunidade de obter mais esclarecimento sobre sua condição de saúde/doença costumam gerar a satisfação tanto pelo atendimento como pela oportunidade de colaborar para o aprendizado prático dos estudantes ${ }^{3}$.

A percepção dos pacientes acerca do atendimento recebido durante as sessões de fisioterapia varia segundo o caráter individual e pode não coincidir com as normas e orientações adotadas pela bioética e ética profissional, gerando diversidade ou até mesmo divergência de interpretação. A esse respeito, percebe-se que poucas são as pesquisas que estudaram os conflitos morais na prática profissional e no ensino da fisioterapia 4 .
As Diretrizes Curriculares do Curso de Fisioterapia recomendam que os graduandos de fisioterapia obtenham conhecimentos sobre comunicação, tomada de decisão, princípios da ética e bioética, bem como desenvolvam habilidades para atuar de forma multiprofissional, interdisciplinar e transdisciplinar, incentivando e justificando que se investigue como isso ocorre na prática profissional, segundo a percepção dos pacientes ${ }^{5}$.

O Código de Ética e Deontologia da Fisioterapia (CEDF) traz importantes orientações para a relação fisioterapeuta-paciente, sobretudo sobre os temas autonomia, remuneração profissional e sigilo ${ }^{6}$. No entanto, o contínuo advento de inovações promove o aparecimento de novas situações moralmente conflituosas que suscitam a necessidade de ampliar o leque de orientações aos profissionais.

Diante disso, o objetivo do estudo foi avaliar a percepção dos pacientes acerca dos aspectos morais na inter-relação entre pacientes, estudantes e fisioterapeutas na assistência fisioterápica em uma universidade do Meio-Oeste catarinense.

\section{MÉTODOS}

Tratou-se de um estudo descritivo, transversal, com abordagem quantitativa, em que foram entrevistados pacientes atendidos por alunos da $8^{\mathrm{a}}$ e $10^{\mathrm{a}}$ fase do curso de fisioterapia, nos estágios curriculares nas áreas de Atenção Básica à Saúde, Fisioterapia Neurofuncional, Musculoesquelética e Hospitalar.

No que diz respeito às questões éticas, inicialmente, foi enviada uma declaração às instituições coparticipantes do estudo que foram: a Coordenação do Curso de Fisioterapia; a Coordenação da Clínica Escola de Pesquisa e Atendimento em Fisioterapia (Cepaf); a Coordenação de Área; o(a) Diretor(a) do Hospital Univer- 
sitário Santa Terezinha e o(a) Secretário(a) Municipal da Saúde. Em seguida, o projeto foi encaminhado ao Comitê de Ética em Pesquisa (CEP) da instituição, o qual, após apreciação, foi aceito mediante o parecer n. 2.578.732. Aos participantes que aceitaram participar do estudo, foi disponibilizado o Termo de Consentimento Livre e Esclarecido (TCLE).

Para a coleta de dados, foi aplicado um questionário estruturado, com 23 perguntas fechadas, sendo que todos os pacientes atendidos nas instituições coparticipantes com a presença de acadêmicos foram convidados a participar. Os critérios de exclusão foram: pacientes que não aceitaram participar da pesquisa; menores de 18 anos; com baixo nível de cognição; desconhecimento da língua portuguesa; em uso de ventilação mecânica ou que se encontravam sedados.

Os assuntos abordados no instrumento de pesquisa foram escolhidos com base nos problemas mais frequentes encontrados na revisão de literatura e com as práticas diárias na fisioterapia, segundo a observação dos próprios autores. A estruturação das questões para cada tipo de percepção ou atitude baseou-se nas orientações contidas na literatura ${ }^{7}$.

A coleta foi realizada nas duas primeiras semanas do segundo semestre do ano letivo de 2018. Para os pacientes atendidos na Cepaf, situada no Ambulatório Universitário (AMU), os questionários foram aplicados pelo pesquisador responsável ou pelos estagiários antes ou depois da sessão de fisioterapia. A aplicação do questionário para os pacientes atendidos externamente à clínica escola (Hospital Universitário, clínica de fisioterapia e domicílio) foi realizada durante os atendimentos pelos estagiários, após receberem o respectivo treinamento.

Os dados foram tabulados em planilha do programa Microsoft Excel ${ }^{\oplus}$, e posteriormente, a análise estatística foi realizada nos softwares IBM SPSS statistics (versão 24.0) ou Le Sphinx Plus2 (versão 4.5), adotando nível de significância quando $\mathrm{p} \leq 0,05$. A existência de diferenças nas prevalências às respostas das questões intragrupos foi analisada por meio do teste de Qui-Quadrado ( $\chi 2)$.

Os resultados serão divididos/descritos em três eixos - perfil dos participantes, atendimento realizado pelos estudantes e conflitos morais -, abordando os temas: uso do TCLE; pudor e intimidade; pacientes HIV/Aids; atração física ou sexual; honorários; e preparo para a vivência de morte.

\section{RESULTADOS}

\section{Perfil dos participantes}

Dos 174 pacientes convidados a fazer parte do estudo, participaram 117, já que 57 se enquadraram em algum dos critérios de exclusão. Prevaleceram os participantes do sexo feminino e da faixa etária entre 45 anos e 60 anos. Quanto à situação conjugal, verificou-se que os pacientes eram predominantemente casados e com o nível de escolaridade fundamental, conforme apresentado na Tabela 1. 


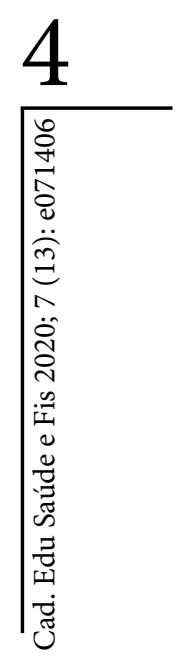

Em relação ao local de atendimento, 65,8\% dos pacientes foram atendidos na Cepaf; $10,2 \%$, no Hospital Universitário; 23,1\%, nos Serviços Municipais de Fisioterapia (grupos e clínica); e 0,9\%, na Home Care, pelo município. Referente ao tempo que estavam recebendo atendimento com a presença de estudantes, 50,4\% eram de até 3 meses; 9,4\%, de 3 a 6 meses; $4,3 \%$, de 6 a 9 meses; 2,6\%, de 9 a 12 meses; e 33,3\% ,de mais de 1 ano; e $0,8 \%$ não respondeu.

Tabela 1. Características sociodemográficas do grupo de pacientes

\begin{tabular}{lcl}
\hline Características dos pacientes & $\mathrm{n}$ & $(\%)$ \\
\hline Situação conjugal & 57 & $48,7 \%$ \\
\hline Casado & 23 & $19,7 \%$ \\
Solteiro & 17 & $14,5 \%$ \\
Separado & 12 & $10,3 \%$ \\
Viúvo & 08 & $6,8 \%$ \\
Outra situação & & \\
\hline Faixa etária & 15 & $12,8 \%$ \\
\hline De 18 a 30 anos & 22 & $18,8 \%$ \\
De 31 a 45 anos & 43 & $36,8 \%$ \\
De 46 a 60 anos & 37 & $31,6 \%$ \\
Mais de 61 anos & & \\
\hline Sexo & 64 & $54,7 \%$ \\
\hline Feminino & 53 & $45,3 \%$ \\
Masculino & & \\
\hline Escolaridade & 04 & $3,4 \%$ \\
\hline Analfabeto & 52 & $44,4 \%$ \\
Ensino fundamental & 43 & $36,8 \%$ \\
Ensino médio & 16 & $13,7 \%$ \\
Ensino superior & & \\
Pós-graduação & & \\
\hline & & \\
\hline
\end{tabular}




\section{Atendimento realizado pelos estudantes}

Questionados acerca da satisfação em contribuir para o aprendizado dos estudantes, 98,3\% dos participantes relataram estar "muito satisfeitos"; e 1,7\% “pouco satisfeitos”. Sobre sentir constrangimento com a presença dos estudantes durante o tratamento fisioterápico, 2,6\% responderam "geralmente"; 7,7\%, "às vezes"; $4,3 \%$, "raramente"; e 85,4\%, "nunca”. Quando indagados se recebiam informações mais claras sobre sua saúde, com a presença de estudantes, 94\% dos pacientes confirmaram; e 6\% deles negaram. Sobre receberem mais informações sobre sua saúde com a presença de estudantes de fisioterapia, em comparação com o médico ou fisioterapeuta isoladamente, $66,7 \%$ responderam "sim"; 31,6\%, "não"; e 1,7\% não respondeu.

Em relação às justificativas apresentadas pelos participantes que demonstraram muita ou pouca satisfação em contribuir para o aprendizado do estudante de fisioterapia, 60 (52,6\%) responderam "Preciso cuidar da minha saúde, e os alunos precisam aprender"; 24 (21,1\%), "Uma oportunidade de convívio social”; 16 (14\%), "Sou mais bem tratado aqui do que em casa"; 7 (6,1\%), “Cansado (a), pois faço tratamento há tempo e não melhoro"; 5 (4,4\%), “outros sentimentos"; e $2(1,8 \%)$ não responderam. Entre os que estavam pouco satisfeitos, 1 (50\%) respondeu "Preciso cuidar da minha saúde, e os alunos precisam aprender"; e 1 (50\%) respondeu "Cansado (a), pois faço tratamento há tempo e não melhoro". Houve 1 (100\%) que não respondeu à pergunta sobre satisfação, mas assinalou a justificativa "Preciso cuidar da minha saúde e os alunos precisam aprender”. Não houve diferença estatisticamente significativa entre as respostas $(\mathrm{p}=0,701)$, de acordo com a tabela 2 .

Tabela 2. Cruzamento das respostas sobre satisfação em estar contribuindo para o aprendizado dos alunos e as justificativas da escolha por grupos de respondentes

\section{Muito Satisfeito Pouco Satisfeito Não Respondeu Total}

\begin{tabular}{|c|c|c|c|c|c|c|c|c|}
\hline Justificativas & $\mathbf{n}$ & $\%$ & $\mathbf{n}$ & $\%$ & $\mathbf{n}$ & $\%$ & $\mathbf{n}$ & $\%$ \\
\hline $\begin{array}{l}\text { Preciso cuidar da minha } \\
\text { saúde, e eles precisam } \\
\text { aprender }\end{array}$ & 60 & 52,6 & 1 & 50,0 & 1 & 100,0 & 62 & 53,0 \\
\hline $\begin{array}{l}\text { Uma oportunidade de con- } \\
\text { vívio social }\end{array}$ & 24 & 21,1 & 0 & 0,0 & 0 & 0,0 & 24 & 20,5 \\
\hline $\begin{array}{l}\text { Sou mais bem tratado aqui } \\
\text { do que em casa }\end{array}$ & 16 & 14,0 & 0 & 0,0 & 0 & 0,0 & 16 & 13,7 \\
\hline $\begin{array}{l}\text { Cansado (a), pois faço } \\
\text { tratamento há tempo e não } \\
\text { melhorou }\end{array}$ & 7 & 6,1 & 1 & 50,0 & 0 & 0,0 & 8 & 6,8 \\
\hline Outro(s) sentimento(s) & 5 & 4,4 & 0 & 0,0 & 0 & 0,0 & 5 & 4,3 \\
\hline Não respondeu & 2 & 1,8 & 0 & 0,0 & 0 & 0,0 & 2 & 1,7 \\
\hline Total & 114 & 100,0 & 2 & 100,0 & 1 & 100,0 & 117 & 100,0 \\
\hline
\end{tabular}


Para a questão se alguma vez, durante consulta ou sessão, o acadêmico de fisioterapia demonstrou desrespeito ou foi antiético com sua pessoa, $0,8 \%$ dos participantes responderam “às vezes"; 5,1\%, "raramente"; e 94,1\%, "nunca". Quando solicitados se deixariam de comunicar alguma informação referente à sua saúde na presença de estudantes, 3,4\% responderam "geralmente"; 13,7\%, "raramente"; 0,9\%, "às vezes"; e $82 \%$, "nunca". Ainda, ao serem questionados sobre a possibilidade de não revelar informações por receio de que os alunos contassem para outras pessoas, $6 \%$ confirmaram, e $94 \%$ negaram.

Os pacientes também foram questionados sobre os alunos se apresentarem como estudantes de fisioterapia e solicitarem seu consentimento antes de realizar o tratamento, encontrando-se $87,2 \%$ que responderam "sim"; $6,0 \%$, “não"; e 7,7\%, “às vezes”. Quanto a terem recebido mais informação sobre o tratamento fisioterápico realizado nos atendimentos, 95,7\% dos pacientes responderam "geralmente"; $3,4 \%$, "às vezes"; e 0,9\%, "raramente".

\section{Conflitos morais}

Avaliando a conduta do fisioterapeuta que pretende expor em um evento científico as fotos do joelho de um paciente sem sua autorização com a justificativa de não ser identificável pelos congressistas, $50,4 \%$ dos participantes responderam “está correto", enquanto 49,6\% responderam "não está correto", conforme apresentado na Tabela 3.
Tabela 3. Percepção dos participantes em relação ao fisioterapeuta apresentar as fotos do joelho de um paciente em evento científico sem sua autorização.

\begin{tabular}{lcc}
\hline & n & $\%$ \\
\hline Está correto & 59 & 50,4 \\
Não está correto & 58 & 49,6 \\
Total & 117 & 100 \\
\hline
\end{tabular}

Em caso de problema ginecológico, urológico ou outro situado em uma parte íntima de seu corpo, $17,1 \%$ responderam que se sentiriam "muito constrangidos" durante o atendimento; $39,3 \%$, "pouco constrangidos"; 42,7\%, "nada constrangido"; e $0,9 \%$ não respondeu.

Acerca do fisioterapeuta ter direito de conhecer a sorologia HIV do paciente, 91,5\% dos pacientes responderam "sim tem direito"; e $8,5 \%$, "não", de acordo com a Tabela 4.

Tabela 4. Percepção dos participantes quanto ao direito do fisioterapeuta em conhecer a sorologia HIV do paciente

\begin{tabular}{lcc}
\hline & $\mathbf{n}$ & $\%$ \\
\hline Sim & 107 & 91,5 \\
Não & 10 & 8,5 \\
Total & 117 & 100 \\
\hline
\end{tabular}

Outrossim, ao fazer a pergunta inversa, se o paciente tem o direito de conhecer a sorologia HIV do fisioterapeuta, 64,9\% dos participantes responderam "sim”; 34\%, "não"; e 1,1\% não respondeu. Sobre sentir atração física ou sexual pelo fisioterapeuta, $0,9 \%$ deles responderam "às vezes"; 1,7\%, "raramente"; e 97,4\%, "nunca". 
Quanto ao fisioterapeuta cobrar pelo atendimento do seu avô que teria condições financeiras para pagar, $40 \%$ dos pacientes responderam "não deve cobrar"; 9\%, "pode não cobrar"; e 51\%, “deve cobrar", como ilustra a Tabela 5.

Tabela 5. Percepção dos participantes em relação ao fato do fisioterapeuta realizar cobrança de honorários de avô

\begin{tabular}{lcc}
\hline & n & $\%$ \\
\hline Não deve cobrar & 47 & 40 \\
Pode não cobrar & 10 & 9 \\
Deve cobrar & 60 & 51 \\
\hline Total & 117 & 100
\end{tabular}

Sobre a importância de o fisioterapeuta estar preparado para vivenciar a morte do paciente, 91,4\% consideraram "muito importante"; 4,3\%, "pouco importante"; e 4,3\%, "nada importante".

\section{DISCUSSÃO}

A faixa etária dos participantes do estudo (Tabela 1) assemelhou-se aos resultados de estudos encontrados em serviços interdisciplinares espanhóis8, em que $42 \%$ tinham mais de 45 anos, embora houvesse menor número de mulheres (42\%), diferindo do presente estudo em que essas eram a maioria (54\%). Em um estudo de serviço de fisioterapia na Inglaterra ${ }^{9}$, também foi constatada a predominância de mulheres $(63,6 \%)$. Quanto ao estado civil e local de atendimento, os dados deste estudo se equivalem ao estudo que verificou a percepção dos pacientes acerca dos estudantes de medicina, sendo que, entre os 196 participantes pesquisados, 64\% eram do sexo feminino, $57 \%$ eram casados e $51 \%$ foram atendidos em um ambulatório universitário de diversas especialidades ${ }^{2}$.

Quanto ao local e tempo de atendimento, percebeu-se que o maior público no âmbito da reabilitação está nos serviços ambulatoriais que abrangem pacientes subagudos e crônicos em diversas especialidades. No caso do presente estudo, a Cepaf é uma clínica escola de pesquisa e atendimento em fisioterapia na qual estão alocados os serviços nas áreas de fisioterapia ortopédica, traumatológica e reumatológica neurológica infantil e adulto, cardiorrespiratória uroginecologia e obstetrícia e oncológica, o que justifica a maior demanda de pacientes.

A maioria mostrou-se satisfeita com o atendimento em que participam os estudantes. No estudo10, foi analisada a percepção dos pacientes sobre o tratamento fisioterápico em centros de reabilitação na Espanha; e, de acordo com suas afirmações, os fisioterapeutas supervisores tinham mais conhecimento acumulado do que outros profissionais da equipe que era composta por fisioterapeutas e médicos. Infere-se, portanto, que, em âmbito acadêmico, os alunos precisam ter um amplo conhecimento das doenças e suas implicações no dia a dia dos pacientes e que a habilidade de comunicação se faz necessária para informar e educar os pacientes sobre sua saúde, uma vez estão sendo monitorados e avaliados constantemente. Assim sendo, essa preocupação com a assistência e, ao mesmo tempo, com a formação dos alunos acaba por se refletir em aprimoramento dos próprios profissionais, bem como no melhor atendimento aos pacientes.

Os pacientes atendidos por acadêmicos da fisioterapia foram questionados se ficavam constrangidos com a presença de estudantes, $\mathrm{e}$ a maioria $(85,4 \%)$ negou, o que pode ser justificado pelas características da população estudada, 
ou seja, de mulheres casadas, bem informadas e mais receptivas às sessões de reabilitação com a presença de acadêmicos. Em contrapartida, os demais pacientes que responderam se sentirem constrangidos em algum momento possivelmente são aqueles atendidos na área de uroginecologia, pois demanda maior exposição das partes íntimas do corpo. Ressalte-se, ainda, que os atendimentos são supervisionados pelos professores fisioterapeutas e são gratuitos, motivos que podem contribuir para melhor aceitação de estudantes durante o tratamento, conforme ficou evidente nesta pesquisa.

Para a maioria dos pacientes, as informações que recebem dos estudantes sobre sua saúde são mais claras e em maior quantidade quando comparadas com o atendimento por médico ou fisioterapeuta individualmente, dados que se assemelham ao estudo ${ }^{8} \mathrm{em}$ que, nos relatos dos pacientes, foi constatado que o cuidado interpessoal em fornecer boa explicação sobre sua doença e/ou prognóstico é fundamental para a boa avaliação do serviço. Como exemplos, apresentam o relato feito por mulher de 48 anos, com fratura de membros inferiores, que elogiava a gentileza dos fisioterapeutas e considerava que os médicos tinham uma atitude mais distante . $^{8}$ Em outro relato, um homem de 37 anos, com lesão ligamentar, considerou as explicações do fisioterapeuta muito importantes para o entendimento dos procedimentos ${ }^{8}$.

Ao relacionar a justificativa do paciente com a satisfação dos pacientes em contribuir para o aprendizado do estudante de fisioterapia, verificou-se que a necessidade de cuidar da saúde foi a resposta mais frequente (Tabela 2). Esses resultados se equivalem ao estudo ${ }^{2}$ em que a maioria dos pacientes se sentia satisfeita ou não se contrapunha à presença de estudantes, pois, em geral, eram atendidos durante um tempo mais prolongado, com mais calma, sendo também uma oportunidade para conversar. Os participantes estão cientes da importância de contribuir para a formação de novos profissionais de saúde, bem como almejam a oportunidade de alcançar melhor nível de saúde e de inter-relação social, corroborando o estudo em que se destaca a importância das circunstâncias sociais, éticas, educacionais e psíquicas presentes nos relacionamentos humanos durante a atenção à saúde ${ }^{11}$.

Em relação a atendimento antiético ou com desrespeito por parte dos acadêmicos, as constatações foram raras e se assemelham a outro estudo $^{2}$ que avaliou a percepção dos pacientes quanto à conduta dos estudantes de medicina durante o atendimento; e somente $10 \%$ relataram episódio de falta de respeito ou educação, índice que deveria ser zero, segundo os autores. Nesse contexto, no estudo que questionou aos docentes fisioterapeutas de uma universidade brasileira se levavam em consideração a postura ética de seus alunos nas avaliações, $94 \%$ responderam que $\operatorname{sim}^{12}$. Por outro lado, no estudo ${ }^{13}$ com os discentes de um curso de fisioterapia, 69,5\% referiram a postura ética como fator integrante das avaliações pelos seus professores. Assim sendo, a postura ética dos acadêmicos perante os pacientes perpassa os preceitos éticos do $\mathrm{CEDF}^{6}$, incluindo-se entre as condutas a serem avaliadas pelos docentes do curso.

Referente a paciente deixar de comunicar algo por constrangimento com a presença de alunos, o resultado também é similar ao estudo no qual $20 \%$ responderam que omitiriam geralmente ou às vezes, mostrando a confiança que a maioria deposita nos estudantes de uma profissão ${ }^{2}$. O sigilo profissional gera situações que estão entre os principais conflitos morais descritos nos estudos nacionais e internacionais. Trata-se de uma relação de confiança entre fisioterapeuta e paciente, em que o compartilhamento das informações é indispensável para o sucesso do trata- 
mento $^{4}$. No CEDF ${ }^{6}$, consta no art. 32, que trata do sigilo profissional, ser proibido ao fisioterapeuta: "Inciso III - fazer referência a casos clínicos identificáveis, exibir cliente/paciente/usuário ou sua imagem em anúncios profissionais ou na divulgação de assuntos fisioterapêuticos em qualquer meio de comunicação, salvo quando autorizado pelo cliente/paciente/usuário ou seu responsável legal". Nota-se, portanto, que o $\mathrm{CEDF}^{6}$ exige expressamente dos profissionais a manutenção do segredo sobre fato sigiloso, em razão da sua atividade profissional.

Segundo os participantes, a maioria dos estudantes apresentou-se e explicou o atendimento que faria. Esses resultados são semelhantes ao estudo no qual $68 \%$ dos fisioterapeutas pediram o consentimento ao paciente antes do tratamento; e 100\% deles explicaram o tratamento para o paciente antes da execução ${ }^{14}$. Porém, em um estudo realizado em Lisboa ${ }^{15}$, mesmo sendo uma obrigação, observou-se que a obtenção do consentimento informado não era uma prática habitual na prática fisioterapêutica, fato que pode influenciar na formação dos acadêmicos.

Em relação à necessidade de obter consentimento do paciente, ao contrário das respostas da maioria dos participantes (Tabela 3), nenhuma imagem pode ser exposta em eventos internos ou externos sem sua autorização prévia, conforme previsto no Inciso II, art. 41 do $\mathrm{CEDF}^{6}$, que orienta obter: "previamente autorização por escrito de cliente/paciente/usuário ou de seu representante legal, por meio de assinatura do termo de consentimento livre e esclarecido para uso de dados, ou no termo próprio de liberação para uso de imagem”. Contudo, essa não foi a percepção dos pacientes.

Os pacientes mostraram-se satisfeitos com as informações recebidas quando estão presentes os estudantes durante os atendimentos. A necessidade de informar aos pacientes sobre a conduta ou qualquer mudança nela necessária durante o tratamento é um dever profissional incluído no $\mathrm{CEDF}^{6}$, em seu art. 10, do capítulo II (responsabilidades fundamentais), em que resta proibido ao fisioterapeuta "recomendar, prescrever e executar tratamento ou nele colaborar, quando praticado sem o consentimento formal do cliente/paciente/ usuário ou de seu representante legal ou responsável, quando se tratar de menor ou incapaz", o que não se refere ao paciente em questão, não justificando a dispensa do consentimento.

Quanto ao direito de conhecer a sorologia de paciente HIV (Tabela 4), como os fisioterapeutas possuem várias áreas de atuação, é possível encontrá-los em clínicas, consultórios, Unidades Básicas de Saúde - UBS e hospitais, entre outros serviços. A chance de contágio por HIV nos ambientes de saúde é mínima, porém, é importante salientar que em clínica que atende pacientes ortopédicos, por exemplo, praticamente não há risco de contágio; todavia, em Unidade de Terapia Intensiva (UTI), onde ocorre contato constante com secreções e sangue, as chances de contágio aumentam. Na literatura ${ }^{16}$, observou-se que o risco de um fisioterapeuta ser infectado por pacientes HIV positivos dificilmente será maior do que para outra pessoa da sociedade de seu contato. Entretanto, cita que mais da metade dos participantes do estudo ${ }^{17}$ e a quarta parte de fisioterapeutas em um estudo ${ }^{18}$ se sentiram em risco de contaminação. Os autores concluem que, por ser mínimo o risco de infecção com as precauções universais instituídas, tal condição não garante a revogação do dever de atendimento dos fisioterapeutas.

Ao examinar as razões ou não razões para o conhecimento do status de HIV de fisioterapeutas e pacientes, autores argumentam ${ }^{19}$ que nenhum desses justifica uma "necessidade de saber", exceto quando a progressão para a Aids ocorreu, e ressalta que os pacientes podem que- 
rer saber se o fisioterapeuta é ou não soropositivo, mas que o tipo de interação terapêutica que ocorre entre os dois cria um risco infinitamente pequeno de transmissão; e, portanto, não haveria justificativa para revogar sua presunção da confidencialidade. No Parecer 11/1992 do Conselho Federal de Medicina (CFM), os relatores discorrem que, mesmo quando o médico é sabidamente infectado, porém não apresenta doença em estado capaz de prejudicar sua competência profissional, considera-se como não obrigatório de sua parte a informação ao paciente sobre sua condição. Também consideraram que a adoção de posição contrária iria prejudicar não só o direito ao trabalho do profissional, como também aumentaria os preconceitos por difundir incorretamente a existência de risco de transmissão do HIV por contato casual ${ }^{20}$.

Percebe-se, portanto, que o assunto não obtém unanimidade de interpretação, o que evidencia a necessidade de mais estudos, sobretudo em âmbito nacional, para ampliar o conhecimento e disponibilizar orientações mais claras sobre as boas condutas dos profissionais que atendem nessas condições. Infere-se que, embora haja indícios de que a maioria não leia ${ }^{21}$, a inserção de orientações no $\mathrm{CEDF}^{6}$ sobre o atendimento em que fisioterapeutas ou pacientes possam estar com afecção pelo HIV contribuiria efetivamente para melhorar a orientação daqueles, refletindo-se em maior sensação segurança para estes.

Pelos dados encontrados sobre assédio sexual, como as respostas positivas sobre sua existência foram muito raras, é possível que possa ter havido algum constrangimento dos participantes em manifestar seus sentimentos, pois geralmente a relação entre profissional, estudante de fisioterapia e paciente é bastante íntima e duradoura. De qualquer forma, infere-se a necessidade de abordagem mais ampla sobre os limites sexuais da relação terapeuta-paciente durante a graduação, bem como a divulgação das orientações constantes no $\mathrm{CEDF}^{6}$ que tratam especificamente sobre o respeito ao pudor para prevenir a inadequação de condutas dos profissionais. Recentemente, em 2018, o Crefito 5 realizou um evento sobre assédio moral e sexual em que foram levantadas as dificuldades de interpretação no âmbito da fisioterapia, considerando-se que o toque faz parte do tratamento e a interpretação de sua realização pode não ser coincidente com a intenção do profissional e ser considerado um abuso. Por outro lado, também pode ensejar a oportunidade para que ocorra o abuso de fato ${ }^{22}$.

Referente aos honorários profissionais, somente a minoria respondeu que pode não cobrar de seu avô (Tabela 5), que é a resposta certa, conforme orientação do art. 38 do $\mathrm{CEDF}^{6}$ que traz importante esclarecimento sobre a possibilidade de o fisioterapeuta deixar de cobrar honorários por assistência prestada somente em três situações: "I - ascendente, descendente, colateral, afim ou pessoa que viva sob sua dependência econômica; II - colega ou pessoa que viva sob a dependência econômica deste, ressalvado o recebimento do valor do material porventura despendido na prestação da assistência; III - pessoa reconhecidamente hipossuficiente de recursos econômicos".

Em relação ao preparo dos fisioterapeutas para a vivência de morte, que os participantes entenderam ser desejável, um estudo ${ }^{23}$ com discentes de medicina em uma universidade do Rio Grande do Sul mostrou que apenas 50\% consideravam-se "preparados", e 47,3\% declararam-se "despreparados" para o enfretamento da morte de pacientes, sendo que, para $54,1 \%$, os principais sentimentos diante dessa situação foram o medo, a incapacidade e o sofrimento. Nesse aspecto, outro autor ${ }^{24}$ observou que a formação do fisioterapeuta é insuficiente no que se refere às 
questões da dimensão espiritual, já que se reveste de cunho mais tecnicista e menos humanista, não valorizando um aspecto de crucial importância que é o final da vida dos pacientes. A experiência da vivência de morte tem proporcionado modificações afetivas na vida da maioria dos acadêmicos de fisioterapia, embora haja carência dessa abordagem durante a formação profissional, o que acarreta o despreparo do profissional para lidar com essa situação ${ }^{25}$.

\section{CONCLUSÕES}

A maioria dos pacientes demonstrou estar satisfeita com o atendimento fisioterápico em que estavam presentes acadêmicos de fisioterapia, bem como em contribuir para seu ensino, sob a justificativa de que recebem mais informações e com mais clareza sobre sua saúde.

Os problemas éticos apontados pelos pacientes durante os atendimentos realizados no âmbito do ensino da fisioterapia são raros e ocorrem, sobretudo, por falta de pedido de consentimento ou por tratamento desrespeitoso dos acadêmicos. Poucos pacientes deixariam de comunicar algum problema de saúde por constrangimento ou por temor de quebra do sigilo profissional, embora a maioria tenha manifestado alguma restrição em caso de procedimentos ginecológicos, urológicos ou em partes íntimas. Em contrapartida, contrariamente às orientações éticas, a maioria entendeu que o fisioterapeuta deve cobrar de seu familiar (avô) e tem direito a conhecer a sorologia HIV do paciente.

Referente à finitude da vida, os pacientes consideraram o preparo para a vivência de morte por parte dos estudantes muito importante. Contudo, sabe-se que o assunto é pouco abordado não só no ambiente profissional, mas também na prática do ensino. Portanto, o assunto morte foi identificado como merecedor de maior atenção durante a formação e a prática profissional, denotando o atendimento humanizado como condição peculiar no cuidado das pessoas/pacientes.

Infere-se que, durante graduação, a oferta de orientações sobre assuntos em que os resultados apontaram problemas éticos no atendimento, como cobrança de honorários, atendimento de pacientes HIV positivo, respeito ao pudor do paciente e conduta respeitosa no relacionamento estudante-paciente, vai contribuir para que a formação dos acadêmicos da instituição seja ainda mais ética e humana, voltada ao cuidado e garantindo uma relação acadêmico/terapeuta/ paciente ainda melhor. Além disso, a inserção de orientações específicas sobre esses temas no CEDF poderá contribuir para aprimorar a relação fisioterapeuta-paciente durante a assistência fisioterápica.

Considerando a limitação e o caráter regional da amostra, novos estudos, com a inclusão de maior número de participantes e instituições, serão necessários para confirmar os resultados encontrados na presente pesquisa, bem como a necessidade da realização de estudos qualitativos para explorar as inquietudes provenientes dos pacientes ante $\mathrm{o}$ atendimento por acadêmicos.

\section{REFERÊNCIAS}

1. Alves FD, Bigongiari A, Mochizuki L, Hossne WS, De Almeida M. O preparo bioético na graduação de Fisioterapia. Fisioter Pesqui. [Internet]. 2008 [citado 2019 jan 28];15(2):14956. Disponível em: http://www.revistas.usp.br/ fpusp/article/view/12058

2. Berwanger J, Denti De Geroni G, Bonamigo EL. Estudantes de medicina na percepção dos pacientes. Rev Bioét [Internet]. 2015 [citado $2018 \mathrm{dez}$ 15];23(3):552-62. Disponível em: http:// 
Heller et al

www.scielo.br/pdf/bioet/v23n3/en_1983-8034bioet-23-3-0552.pdf

3. Carvalho JBS, Moreira Filho RE. Biodireito e bioética: percepções entre fisioterapeutas e estudantes de fisioterapia. RFSF [Internet]. 2014 [citado 2019 jan 15];3(1):18-22. Disponível em: http://www.fisioterapiaesaudefuncional.ufc.br/ index.php/fisioterapia/article/view/383

4. Lorenzo CFG, Bueno GTA. A interface entre bioética e fisioterapia nos artigos brasileiros indexados. Fisioter mov [Internet]. 2013 [citado 2019 jan 15]; 26(4): 763-75. Disponível em: http://www.scielo.br/pdf/fm/v26n4/a06v26n4. pdf

5. Brasil. Resolução CNE/CES no 4 , de 19 fevereiro de 2002. Institui Diretrizes Curriculares Nacionais do Curso de Graduação em Fisioterapia. Diário Oficial da União. 2002 fev 19.

6. Brasil. Conselho Federal de Fisioterapia e Terapia Ocupacional - COFFITO. Resolução n ${ }^{\circ}$ 424, de 8 de julho de 2013. Estabelece o Código de Ética e Deontologia da Fisioterapia,. Diário Oficial da União. 2013 ago. $1^{\circ}$.

7. Vieira S. Como elaborar questionários. São Paulo: Atlas; 2009.

8. Del Baño-Aledo ME, Medina-Mirapeix F, Escolar-Reina P, Montilla-Herrador J, Collins SM. Relevant patient perceptions and experiences for evaluating quality of interaction with physiotherapists during outpatient rehabilitation: a qualitative study. Physiotherapy [Internet]. 2014 [cited 2018 Jan 17]; 00(1):73-79. Available from: https://www.physiotherapyjournal.com/article/ S0031-9406(13)00052-7/fulltext

9. Bishop FL, Smith R, Lewith GT. Patient preferences for technical skills versus interpersonal skills in chiropractors and physiotherapists treating low back pain. Fam Pract [Internet].
2012 [cited 2019 Jan 10];30(2):197-203. Available from: https://academic.oup.com/fampra/ article/30/2/197/499700

10. Medina-Mirapeix F, Oliveira-Sousa S, Sobral-Ferreira M, Baño-Aledo MED, Escolar-Reina P, Montilla-Herrador J, et al. Continuity of rehabilitation services in post-acute care from the ambulatory outpatients' perspective: a qualitative study. J Rehabil Med. 2011 Jan;43(1):58-64.

11. Fortes PAC. Ética, direitos dos usuários e políticas de humanização da atenção à saúde. Saúde e Soc [Internet]. 2004 [citado $2018 \mathrm{dez}$ 10];13(3):30-35. Disponível em: http://www. scielo.br/pdf/sausoc/v13n3/04.pdf

12. Magalhães ÁB, Pereira MNS, Nascimento BNP, De Lima MDS, Gimenes RO, Da Costa Teixeira R. Percepção, interesse e conhecimento de docentes de fisioterapia sobre a ética na profissão. Rev bioét. 2016 [citado 2019 jan 15];24(2):322-331. Disponível em: http://revistabioetica.cfm.org.br/index.php/revista_bioetica/ article/view/1295/1503

13. Coelho PHS. Ética em fisioterapia: interesse, percepção e níveis de conhecimento entre alunos do curso de fisioterapia em uma universidade no interior da Amazônia brasileira [trabalho de conclusão de curso]. Santarém (PA): Universidade do Estado do Pará; 2014.

14. De Araújo LZS, Júnior WAN. A bioética e a fisioterapia nas unidades de terapia intensiva. Fisioter Pesqui [Internet]. 2003 [citado $2018 \mathrm{dez}$ 20];10(2):52-60. Disponível em: http://www. revistas.usp.br/fpusp/article/view/78115/82203 


\section{$\frac{13}{\text { Heller et al }}$}

15. Viegas Soares PAA, Silva CA. Consentimento Informado em Fisioterapia - que realidade? [dissertação]. Évora (LX): Universidade de Évora e Escola Superior de Tecnologia da Saúde de Lisboa; 2008.

16. Voors M. The Duty to Treat: Ethics and HIV/AIDS. Physiotherapy. 2000;86(12):640-644.

17. Sheen D, Green A. Are you positive?: AIDS, attitudes and physiotherapy. Physiotherapy. 1997;83(4):190-196.

18. Dike L. Physiotherapists' perceptions of risk of HIV transmission in clinical practice. Physiotherapy.1993;79(3):178-185.

19. Sim J. Confidentiality and HIV status. Physiotherapy. 1997;83(2):90-96.

20. Brasil. Conselho Federal de Medicina, Assunto: AIDS e Ética Médica [Internet]. Parecer 11 de 1992 [citado 2018 dez 12]. Disponível em: https://sistemas.cfm.org.br/normas/visualizar/ pareceres/BR/1992/11

21. Sousa TS, Silva JS, Silva CFR, Amoras ASL, Silva Neto JR, Picanço KRT, et al. Aplicabilidade dos aspectos éticos no cotidiano do profissional fisioterapeuta: um estudo piloto. Cad Educ Saúde Fisioter [Internet]. 2017 [citado 2018 dez 10];4(8 -Supl.). Disponível em: http://revista. redeunida.org.br/ojs/index.php/cadernos-educacao-saude-fisioter/article/view/1779

22. Debate discute assédio no ambiente de trabalho. Rio Grande do Sul (RS): CREFITO 5 [Internet]; 2018 [citado $2018 \mathrm{dez} 10$ ]. Disponível em: http://www.crefito5.org.br/?p=19433\#. XDHknPZFyps

23. Azeredo NSG. O acadêmico de medicina frente à morte: questões para se (re) pensar a formação [dissertação]. Porto Alegre (RS): Universidade Federal do Rio Grande do Sul-UFRGS; 2007.
24. Mavigner Neto ADP. Percepção da espiritualidade por fisioterapeutas em uma UTI: Uma pesquisa qualitativa [dissertação]. São Leopoldo (RS): Escola Superior de Teologia - EST; 2016.

25. Mizuno YA. Experiências frente à da morte em graduando de fisioterapia: perfil sociodemográfico [trabalho de conclusão de curso]. Brasília (DF): Universidade de Brasília; 2016.
Recebido: 28/02/2019 Aprovado: 21/05/2020 